\title{
El legado materialista de Heidegger
}

\author{
ROBERTO RUBIO* \\ Universidad Alberto Hurtado (Chile) \\ rorubio@uahurtado.cl
}

\begin{abstract}
Resumen
A fines del siglo pasado, con especial notoriedad en los años 80 y 90, surgieron diversos intentos de revuelta anti-hermenéutica que propusieron una transformación materialista de las Ciencias sociales y las Humanidades. Resulta especialmente llamativo el hecho de que algunas de las propuestas más influyentes en ese contexto hayan recurrido explícitamente al pensamiento de Martin Heidegger. En efecto, tanto Hans-Ulrich Gumbrecht cuanto Friedrich Kittler reconocen a Heidegger como una de sus principales influencias. ¿Cómo es posible que el pensamiento de Heidegger, un referente de la filosofía hermenéutica, se haya convertido en un eficaz apoyo para la revuelta contra la orientación hermenéutica de las Ciencias del Espíritu? Esta cuestión exige, por una parte, tomar en consideración el contexto académico de la época, especialmente las tensiones intergeneracionales y el surgimiento de nuevas orientaciones interdisciplinares. Por otra parte, la cuestión reclama una tarea de indagación temática. Es preciso explicitar qué planteamientos de problemas y qué desarrollos específicos provenientes de Heidegger fueron objeto de una recepción creativa conducente a un rediseño materialista de las Ciencias del Espíritu. Palabras clave: Heidegger, materialismo, Ciencias del Espíritu, Gumbrecht, Kittler.
\end{abstract}

\section{Heidegger's materialistic legacy}

\begin{abstract}
Diverse anti-hermeneutical attempts that proposed a materialist transformation of the Social Sciences and the Humanities developed in the late 20th century, particularly during the 80 s and 90s. It is striking that some of the most influential proposals in that context explicitly appealed to Martin Heidegger's thought. In fact, Hans-Ulrich Gumbrecht and Friedrich Kittler acknowledged Heidegger as one of their main influences. How is it possible that Heidegger's thought, a well-known referent of hermeneutics, became a productive resource for the revolt against the hermeneutical orientation of the 'Geisteswissenschaften'? On the one hand, this question requires us to consider the academic context of that time, especially the intergenerational tensions and the birth of new interdisciplinary orientations. On the other hand, it demands a thematic inquiry. We need to explain what problems and specific developments of Heidegger's thought were interpreted in a materialist fashion in order to reshape the 'Geisteswissenschaften'.
\end{abstract} Key words: Heideggger, materialism, Geisteswissenschaften, Gumbrecht, Kittler.

* Doctor en Filosofía por la Ludwig-Alberts Universität Freiburg (2005). Es profesor asociado en la Universidad Alberto Hurtado (Chile), donde actualmente dirige el Departamento de Filosofía. Es autor de Zur Möglichkeit einer Philosophie des Verstehens. Das Produktive Scheitern Heideggers (2006).

Recibido: 6/Marzo/2019 - Aceptado: 3/Mayo/2019 


\section{INTRODUCCIÓN}

El escenario de las Ciencias del Espíritu en Alemania a fines del siglo pasado, con especial notoriedad en los años 80 y 90, estuvo marcado por la confrontación con la Hermenéutica, uno de los paradigmas dominantes. Diversos intentos de revuelta anti-hermenéutica asumieron el carácter programático de una transformación materialista de las Ciencias del Espíritu.

Resulta especialmente llamativo el hecho de que algunas de las propuestas más influyentes en ese contexto de revuelta hayan recurrido explícitamente al pensamiento de Martin Heidegger. En efecto, tanto HansUlrich Gumbrecht, con el proyecto de las Materialidades de la comunicación (Gumbrecht \& Pfeiffer, 1988: 15 sg.; Gumbrecht, 2004: 28-32; 2012: 160 sg., 185-189.), decantado luego en la propuesta de una "filosofía de la presencia" (Gumbrecht, 2007), cuanto Friedrich Kittler, con su propuesta de "expulsar al Espíritu de las Ciencias del Espíritu" (Kittler, 1980), cristalizada en el Materialismo de teoría de la información ${ }^{1}$, reconocen a Heidegger como una de sus principales influencias (Gumbrecht, 2004: 75 sg., 85-98; 2007: 35 sg.; Kittler, 2000: 228-249; 2009a; 2013: 290-302). Con vistas a ello, es posible hablar de una herencia o legado materialista de Heidegger.

Ahora bien, ¿cómo es posible que el pensamiento de Heidegger, un referente de la filosofía hermenéutica, se haya convertido en un eficaz apoyo para la revuelta contra la orientación hermenéutica de las Ciencias del Espíritu? Esta cuestión exige, por una parte, tomar en consideración el contexto académico de la época, especialmente las tensiones intergeneracionales y el surgimiento de nuevas orientaciones interdisciplinares. Por otra parte, la cuestión reclama una tarea de indagación temática. Es preciso explicitar qué propuestas provenientes de Heidegger fueron objeto de una recepción creativa conducente a un rediseño materialista de las Ciencias del Espíritu.

Ambas tareas ocuparán los partes primera y segunda de este trabajo, respectivamente. A modo de conclusión, se propondrá un análisis crítico de tal recepción materialista de Heidegger.

1 Kittler, 1993: 182. Las traducciones de los pasajes citados corresponde al autor del presente artículo. 


\section{LA RECEPCIÓN MATERIALISTA Y ANTI-HERMENÉUTICA DE HEIDEGGER} EN CONTEXTO

La peculiar combinación de materialismo y pensamiento heideggeriano que surgió durante los años 80 y 90 del siglo pasado en Alemania no tuvo lugar al interior de las corrientes de inspiración marxista o neo-marxista, ni tampoco fue una ramificación de la scholarship heideggeriana. No ocurrió al interior de la disciplina filosófica, sino en un ambiente interdisciplinario, organizado principalmente en torno a la conexión entre la teoría literaria y la incipiente ciencia de los medios y marcado por una actitud oscilante de aproximación y distanciamiento respecto a la filosofía.

Las figuras más influyentes en esa escena fueron Hans-Ulrich Gumbrecht y Friedrich Kittler. Poniendo el foco en ellos, se analizará a continuación el contexto de surgimiento de la recepción materialista de Heidegger que aquí nos ocupa.

El primer elemento de contexto a considerar es la situación de conflicto y relevo generacional que tuvo lugar en las universidades alemanas, particularmente en el ámbito de las Ciencias del Espíritu, durante los años 80. Los casos de Gumbrecht y Kittler ilustran dos modalidades de esa situación. Por una parte, hubo una reacción contra la estructura jerárquica universitaria, entre cuyas expresiones más notorias se encontraba la manera de renovar los puestos académicos superiores. Esta consistía en la sucesión de un maestro por un discípulo destacado que alcanzaba así el estatus de continuador. Muchos jóvenes profesores que se sentían excluidos de ese sistema canalizaron su frustración en un rechazo a las Ciencias del Espíritu como proyecto institucional. En este sentido, Gumbrecht habla de una "revuelta edípica" (2004: 26) y pone como ejemplo su propio caso como discípulo de Hans Robert Jauss en la Universidad de Constanza: "el punto de referencia esencial de las revueltas intelectuales es al parecer la generación de nuestros predecesores inmediatos" (2007: 27); "Pero más que contra Jauss, mi inmediata agresión y energía vengadora se dirigieron contra los términos "dialéctica" y "hermenéutica"- y para siempre en verdad, como hoy lo sé” (2007: 30).

Por otra parte, intentos innovadores respecto a metodologías y campos de estudio tuvieron serias dificultades para ser reconocidos, lo cual puso en evidencia la rigidez institucional ante nuevos enfoques y la falta de comprensión respecto a los estilos y propuestas de las nuevas generaciones de académicos. Testimonio de ello es el caso de la habilitación de Friedrich Kittler en la Universidad de Friburgo, cuyo proceso se extendió dos años, de 1982 a 1984, y requirió trece evaluaciones de la tesis, así como la redacción de un prólogo por parte de Kittler a petición de la Comisión 
Examinadora ${ }^{2}$. En relación con ello, afirma Manfred Schneider, un miembro de dicha Comisión, en un texto de 2012: "El Dossier de las evaluaciones y del prólogo añadido por Friedrich Kittler a pedido de la Comisión de Habilitación permite estudiar cómo se impuso el nuevo paradigma científico, hace casi treinta años, a pesar de la fuerte resistencia en la institución académica" (Pias, 2012: 192).

Así como Gumbrecht testimonia los conflictos intergeneracionales en torno a un sistema verticalista y a un estilo casi dinástico de renovación de puestos académicos, el caso Kittler pone de relieve las tensiones intergeneracionales ante el surgimiento de planteos radicalmente innovadores ${ }^{3}$.

El segundo elemento de contexto es el ambiente de discusión en las Ciencias del Espíritu respecto a su idea fundacional y su futuro. En los años 80 y 90 se vive una peculiar tensión en la escena académica alemana: en el momento en que la Hermenéutica, con figuras como Gadamer, Jauss y Koselleck, goza de actualidad internacional y es recibida incluso como la koiné o lengua común de las humanidades y las ciencias sociales ${ }^{4}$, surgen reacciones que apuntan a reconfigurar el proyecto mismo de las Geisteswissenschaften. En ese marco, el materialismo se convirtió en la divisa de la revuelta contra la Hermenéutica en cuanto paradigma fundacional y dominante en las Ciencias del Espíritu y junto con ello en la clave para la transformación de dichas ciencias.

Se trata, pues, en este caso, de un materialismo gestado en relación con la Hermenéutica y con un carácter claramente programático, orientado a la transformación y renovación de las Ciencias del Espíritu. En este sentido, la propuesta de Kittler de "expulsar al Espíritu de las ciencias del Espíritu" (1980) funge como la proclama que orienta su Materialismo de teoria de la información (Kittler, 2013: 324). Por su parte, Gumbrecht caracteriza el proyecto de las Materialidades de la comunicación como parte de una revuelta contra la Hermenéutica, cuya finalidad no es otra que renovar las Ciencias del Espíritu:

Los conceptos "comunicación" y "materialidad" parecían prometer una alternativa al carácter interminable de la interpretación y a la narración del pasado, realizada una y otra vez de nuevos modos (...) Algunos de nosotros

2 Un extracto de las actas y documentos del proceso de habilitación, editado por Claus Pias, fue publicado en 2012, in memoriam Friedrich Kittler, quien falleció el 18 de octubre del 2011. Ver Pias, 2012.

3 Cabe precisar aquí que Kittler, al igual de Gumbrecht, fue asistente de un reconocido germanista cercano a la Hermenéutica. Así como Gumbrecht fue asistente de Hans-Robert Jauss en Constanza, Kittler lo fue de Gerhard Kaiser en Friburgo.

$4 \quad$ En su artículo "Hermenéutica: nueva koiné", Vattimo (1991: 55 sg.) caracteriza a la Hermenéutica como el lenguaje común de la filosofía y la cultura en los años 80. 
queríamos la cultura más sobria de la descripción compleja, cuya eficacia observábamos en las ciencias naturales. (Gumbrecht, 2004: 23)

La estrategia preferente del enfoque materialista anti-hermenéutico fue la relativización de la Hermenéutica mediante un procedimiento genealógico. Así, Gumbrecht (2012: 190 sg.) ofrece una genealogía orientada a mostrar el surgimiento del paradigma hermenéutico, diagnosticar su final y junto con ello sacar a luz el encubrimiento de la dimensión no-hermenéutica. En este sentido, afirma: "Solo es posible ver y reconocer el final histórico de la centralidad cultural y científica de la interpretación si establecemos el inicio histórico de ese estatus. Cuatro etapas (organizadas "genealógicamente" al estilo de Michel Foucault) describirán el camino de mi argumentación" (Gumbrecht, 2012: 191).

Por su parte, en su Tesis de Habilitación, Kittler analiza el surgimiento y desarrollo de la literatura alemana del siglo XIX mediante el estudio de lo que él denomina "sistemas de inscripción", esto es, "redes de técnicas e instituciones que permiten la producción y gestión de datos relevantes en una cultura" (Kittler, 1985: 519). La reconstrucción histórica que Kittler ofrece apunta no solo a desmitificar la literatura (y filosofía) alemana del s. XIX, mostrando sus condiciones técnico-materiales de surgimiento. Junto con ello, se propone echar por tierra el predominio de la Hermenéutica como paradigma en las Ciencias del Espíritu. En este sentido, en la introducción a la traducción al inglés de la Tesis de Habilitación de Kittler, David Wellbery afirma:

Kittler, atendiendo no menos intensamente que Nietzsche al poder de la letra, quita el velo de la hermenéutica y disipa su aura, su brillante sugestión de sacral autoridad. Este desmantelamiento de la hermenéutica sigue dos líneas argumentativas entrelazadas. La primera de ellas es histórica, o dicho con mayor precisión: genealógica, en el sentido nietzscheano del término. En la perspectiva del análisis genealógico, la pretensión de universalidad de la hermenéutica se evapora y la hermenéutica queda expuesta en su ligazón a la situación, en su particularidad. (Wellbery, 1990: ix-x)

Respecto a la genealogía de la Hermenéutica que Kittler ofrece cabe aplicar algo que el propio Kittler atribuye a lo que él denomina la "genealogía de la literatura" realizada por Nietzsche, a saber: que ella "describe el surgimiento y la declinación" de su objeto (Kittler, 2013: 25).

Corresponde además realizar aquí una precisión. En diversas ocasiones, Gumbrecht insiste en aclarar que su postura no es anti-hermenéutica, sino que apunta más bien a rehabilitar elementos no-hermenéuticos y un enfoque que les haga justicia, sin excluir con ello la dimensión del sentido 
y la interpretación (Gumbrecht, 2004: 18; 2012: 191). En esto, Gumbrecht contrasta claramente con las propuestas más radicales de Kittler, quien insta a eliminar la noción misma de sentido como criterio de análisis (Kittler, 2010: 44). Por otra parte, sin embargo, Gumbrecht rechaza la pretensión de universalidad de la Hermenéutica ${ }^{5}$ y objeta el rol de la Hermenéutica como paradigma dominante de las Ciencias del Espíritu (Gumbrecht, 2004: 12, 17 sg.; 2012: 160 sg., 185 sg.). En este punto se puede hablar sin duda de un gesto anti-hermenéutico en Gumbrecht, coincidente con el de Kittler. Más aun, Gumbrecht (2004: 24 sg.) refiere expresamente a la influencia de Kittler en el ambiente de revuelta contra el predominio de la Hermenéutica durante los años 80. Cabe mencionar también que la influencia de Kittler en el pensamiento de Gumbrecht fue constante y dio lugar a vínculos de amistad y trabajo colaborativo ${ }^{6}$.

Un tercer elemento de contexto es la recepción del postestructuralismo ${ }^{7}$. Durante los años 70 y 80 tuvo lugar en Alemania una creciente apertura, cargada de conflictos y resistencias, a los planteos de Foucault, Lacan, Derrida y Deleuze, entre otros. Tales planteos constituían una combinación especialmente difícil de asimilar: por una parte, representaban una de las líneas más punzantes de la revolución generacional y cultural de mayo de 1968, por otra parte, traían consigo de regreso a Alemania el peso de la tradición filosófica local, con nombres como Hegel, Marx, Nietzsche y Heidegger. Como afirma Garnica (2018: 47): "Lo propio de la filosofía alemana del siglo XIX y XX retorna en los planteamientos de la filosofía de Mayo del 68".

"Integración sin entusiasmo": tal es la fórmula que acuña Manfred Frank (2004: 5) para describir la manera en que se abordó en Alemania el contacto con el propio pasado filosófico provocado por la recepción del pensamiento francés, particularmente el post-estructuralismo. Ahora bien,

$5 \quad$ Ver Gumbrecht, 2004: 17-18; 75 sg. Tal pretensión fue popularizada especialmente a partir de Gadamer (1986: 478 sg.; 1999: 567 sg.). Los planteos gadamerianos, difundidos y amplificados por filósofos y teóricos de otras disciplinas humanísticas, provocaron agudas discusiones en el campo de la epistemología de las ciencias sociales y humanas, especialmente entre los años 70 y 90 -ver especialmente Habermas (1970). En el contexto de esas discusiones se inscribe el clima de transformación y renovación en el cual se sitúan las tendencias materialistas que aquí analizamos.

6 Así, Kittler publicó un artículo en el volumen "Materialidades de la comunicación", editado por Gumbrecht y Pfeiffer en 1988. Por su parte, Gumbrecht editó y escribió el postfacio de la compilación de textos de Kittler, publicada el año 2013 bajo el título Die Wabrheit der technischen Welt (La verdad del mundo técnico).

7 El término "postestructuralismo" no carece de dificultades y polémicas. Cabe recordar aquí que tal denominación no fue propuesta ni aceptada completamente por las y los pensadores a quienes se aplicó. Para una consideración más detallada del postestructuralismo francés, ver Dillet et al., 2013. 
dicha fórmula refleja más bien la actitud del propio Frank, quien fue una figura activa en la recepción y difusión del postestructuralismo, aunque ejerciendo un distanciamiento crítico. ${ }^{8}$ En términos generales, las líneas predominantes en Filosofía y Humanidades en Alemania durante los años 80, esto es, la Teoría Crítica y la Hermenéutica, fueron muy poco integradoras (Winthrop-Young y Wutz, 1999: xvi-xviii). Las críticas enérgicas de Habermas (1985: 1993), por una parte, y el controvertido (des)encuentro entre Gadamer y Derrida ${ }^{9}$, por otra, testimonian tal situación.

El postestructuralismo, con su énfasis en los aspectos opacos e inconscientes de la cultura, su crítica a la tradición metafísica y a la filosofía moderna centrada en el sujeto, su radicalización del procedimiento genealógico y su especial interés en la literatura, influyó decisivamente en un conjunto de jóvenes investigadores alemanes, particularmente del ámbito de la Ciencia de la Literatura. El pathos rupturista y revolucionario del postestructuralismo, que había sido rechazado frontalmente o bien relativizado por las corrientes dominantes de las Ciencias del Espíritu, tuvo especial acogida entre las nuevas generaciones de académicos que procuraban una transformación general de dichas ciencias. En este sentido, Gumbrecht (2004: 27) reconoce para sí y para quienes compartían su "intención edípico-revolucionaria", que "continuábamos queriendo ser los portadores de la antorcha de 1968". Por su parte, el texto de habilitación de Kittler, publicado en 1985, significó la aplicación creativa y personal de planteos postestructuralistas, especialmente de Foucault y Lacan, para la revuelta anti-hermenéutica al interior de las Ciencias del Espíritu. En relación con ello, afirma Wellbery:

Redes de Discurso [Aufschreibesysteme 1800/1900. / RR.] revela de una manera más clara que lo ocurrido hasta ahora en Estados Unidos, que un criticismo literario informado por el postestructuralismo es, de hecho, un criticismo posthermenéutico. Tal criticismo abandona el juego de lenguaje y la forma de vida definida por los cánones hermenéuticos de justificación e ingresa en dominios de investigación inaccesibles para los actos de comprensión apropiativa. (Wellbery 1990, ix)

8 Frank propone redefinir al así llamado Post-estructuralismo como Neoestructuralismo, matizando así el pretendido carácter superador y rupturista atribuido a aquel. Ver Frank, 2011. Respecto a las oscilaciones de Frank en su consideración del pensamiento francés del 68, ver Garnica, 2018.

$9 \quad$ En abril de 1981 tuvo lugar en el Instituto Goethe en París un encuentro entre Gadamer y Derrida, con ocasión del Coloquio “Texto e Interpretación” organizado por Philippe Forget. Las respuestas recíprocas a las ponencias de ese Coloquio fueron publicadas en 1984 en la Revue internationale de philosophie. En general, las impresiones del organizador, de los participantes y de la crítica fue que no tuvo lugar allí un genuino debate. Ver Michelfelder \& Palmer, 1989: 4 sg. 
Un último elemento de contexto a considerar es el desarrollo de la incipiente Ciencia de los Medios (Medienwissenschaft) en Alemania durante los años 70 y 80 . Como documenta Claus Pias, los referentes para la primera generación de cientistas de los medios provenían desde fuera, sea desde la filosofía, la historia o la teoría literaria: "los scholars de los medios no podrian haber inventado el estudio ni la scholarship de los medios" (Pias, 2016: 22). Esta situación generó un particular ambiente interdisciplinar, abierto a influencias diversas. En este contexto específico, la influencia materialistatecnicista de McLuhan y la Escuela de Toronto, muy resistida inicialmente por los teóricos de los medios cercanos a la Escuela de Frankfurt ${ }^{10}$, fue especialmente acogida por los jóvenes investigadores abiertos al postestructuralismo, como Gumbrecht y Kittler ${ }^{11}$.

Presentados ya estos tres elementos de contexto, corresponde ahora mostrar cómo influyeron en la recepción materialista de Heidegger. En primer lugar, hay que destacar que tal recepción tuvo lugar inmediatamente tras la muerte de este y por impulso de la acogida que tuvo su pensamiento fuera de Alemania. Ello explica que la figura de Heidegger no haya sido el blanco directo de las energías revolucionarias-edípicas a las que Gumbrecht refiere, pues estas se dirigían especialmente a la generación de los Professoren en ejercicio, como por ejemplo Jauss ${ }^{12}$. Además, la recepción post-estructuralista de Heidegger situó a este en el contexto de la revuelta generacional del mayo francés, con lo cual el reingreso de Heidegger a Alemania estuvo signado por un peculiar aggiornamento, no exento de polémicas.

En segundo lugar, hay que considerar que la recepción postestructuralista de Heidegger puso de relevancia ciertos planteamientos que encontraron cabida rápidamente en la agenda del materialismo anti-hermenéutico. Entre estos destacan la crítica a la Metafísica (enfocada particularmente en la vinculación entre Metafísica y Modernidad), el procedimiento

10 Un caso paradigmático es el de Hans Magnus Enzensberger. Sobre la posición de Enzensberger, ver Mersch, 2006: 73-78. Sobre la crítica de Enzensberger a McLuhan, ver Enzensberger, 1982: 66-68; Pias, 2016: 24; Winthrop-Young \& Wutz, 1999: xiv-xvi.

11 Ver Gumbrecht, 2004: $28 \mathrm{sg}$. Sobre la influencia del postestructuralismo francés en Kittler, ver Winthrop-Young \& Wutz, 1999: xvi-xviii.

12 El propio Gumbrecht pone de relieve su actitud diferenciada, en un caso respecto a Jauss, y en otro respecto a Heidegger: "¿Pero no es una contradicción que mi postura vis à vis con el pasado de Jauss haya sido tan agresiva y ofensiva si hoy estoy efectivamente contaminando a las futuras generaciones intelectuales con el pensamiento de un filósofo que estuvo tipológicamente más próximo de la ideología nacionalsocialista (...)? (...) Lo único que importa en el contexto de este ensayo es el hecho de que el punto de referencia esencial de las revueltas intelectuales es al parecer la generación de nuestros predecesores inmediatos" (2007: 27). 
de la destrucción, cercano a la genealogía y a la deconstrucción, y los planteos anti-humanistas.

Cabe mencionar, en tercer lugar, que bajo la influencia de la teoría de los medios de inspiración mcluhaniana surgió un especial interés por esclarecer el rol de los procesos técnicos en la generación de sentido. Al respecto, afirma Gumbrecht (2004: 28): "encontrábamos especialmente fascinante la cuestión de cómo diversos medios - diversas "materialidades" - de comunicación podían tener efecto sobre el sentido portado por ellos". Este énfasis materialista y tecnicista fue amalgamado con los planteos postestructuralistas, haciendo frente común contra la Hermenéutica. El pensamiento de Heidegger, reingresado a través del postestructuralismo, ofrecía elementos conceptuales y metodológicos para lograr esa amalgama y se convirtió entonces en un eficaz apoyo en la cruzada anti-hermenéutica. De ello nos ocuparemos a continuación.

\section{AsPectos TEMÁticos FUNDAMENTALES}

Para analizar la apropiación materialista de Heidegger de los años 80 y 90 en sus aspectos temáticos, es preciso tener en cuenta sus objetivos y alcances. Como ya se ha mencionado, se trata de un materialismo surgido al interior de las Ciencias del Espíritu y dirigido a reconfigurarlas. Tal reconfiguración es vista como la superación de una estructuración dualista, sostenida por el enfoque hermenéutico dominante. Gumbrecht (2012: 202) plantea esto del siguiente modo: la perspectiva hermenéutica, fundadora de las Ciencias del Espíritu, propone un "campo hermenéutico" articulado en dos ejes: "el eje vertical, la dicotomía entre la 'superficie meramente material' y "la profundidad conceptual"” y "el eje horizontal (...), la excentricidad del sujeto observador frente al mundo de los objetos". A estos dualismos cabe añadir la dicotomía entre Ciencias del Espíritu y Ciencias de la Naturaleza. En tal sentido, para Gumbrecht, el nacimiento de las Ciencias del Espíritu implica programáticamente el distanciamiento respecto a las prácticas y especialmente a las exigencias de las Ciencias Naturales:

Podemos considerar la invención de la asociación de disciplinas llamada "Ciencia del Espíritu" (...) como la salvación del campo hermenéutico al precio de un doble aislamiento. En cuanto ciencia, este tipo de experiencia de mundo queda aislada de la vida cotidiana, y en cuanto ciencia del Espíritu, se libera, dentro del mundo académico, de la presión (...) de tener que compatibilizar su propio saber experiencial con las observaciones del mundo realizadas en las ciencias naturales. (Gumbrecht, 2012: 203) 
Para Gumbrecht, al igual que para Kittler, se impone la tarea de deshacer los dualismos mencionados y de reestructurar las Ciencias del Espíritu. Gumbrecht toma de Kittler un argumento de corte historicista, según el cual la superación de tales dualismos es un proceso en marcha gestado por las transformaciones técnicas en los medios de comunicación. Tales transformaciones habrían conducido al surgimiento de disciplinas que se encontrarían fuera del marco dualista hermenéutico, como la Psico-física del siglo XIX:

Gramófono y Film hacen posible por primera vez el registro y circulación de la percepción, sin que haya que pagar por ello el precio de eliminar los cuerpos percipientes o paralizar en conceptos el movimiento percibido. Friedrich Kittler ha analizado históricamente los efectos de estos dispositivos, en los cuales desde la época en torno a 1900 convergieron, bajo el nombre de "Psicofísica", la corporalidad de la percepción y la psicología de la experiencia. $(2012,201)^{13}$

Conforme a la reconstrucción histórica propuesta por Kittler, los avances en las tecnologías de las comunicaciones durante el siglo XX, particularmente mediante el desarrollo de la computación, condujeron a la superación de la oposición entre lo físico y lo lógico, entre la materia y el sentido: "El sueño de toda la vida de Heidegger, consistente en destruir la oposición binaria entre forma y materia, se podría alcanzar con sencillez con la ayuda de la matemática y la ciencia computacional" (Kittler, 2009a: 29).

Ahora bien, tanto el diagnóstico acerca de los dualismos consolidados en la Hermenéutica cuanto los planteos sobre el proceso de su superación se articulan desde un marco teórico construido en gran medida a partir de la apropiación de Heidegger. El Heidegger que reingresa a Alemania en los años 80, impulsado por el postestructuralismo, ofrece ante todo planteos metodológicos y un repertorio conceptual que permiten articular la estrategia central del materialismo anti-hermenéutico, la cual consiste en elaborar un diagnóstico del presente que desmonte genealógicamente las Ciencias del Espíritu y que ponga de relieve los elementos fundamentales encubiertos y tergiversados por estas. Veamos esto con detalle ${ }^{14}$.

13 Con respecto a la Psicofísica, ver Kittler, 1985: 259-287.

14 Cabe realizar aquí la siguiente aclaración: algunos de los aspectos de la estrategia genealógica que atribuiré a Heidegger pueden encontrarse con diversos énfasis en otros autores como Nietzsche, Foucault o Derrida. Sin embargo, más allá de coincidencias o familiaridades parciales, los elementos que destacaré, considerados en su articulación, corresponden prioritariamente a Heidegger. 
La mencionada genealogía de las Ciencias del Espíritu —y con ello de la Hermenéutica- puede reconstruirse a través de los siguientes planteos fundamentales:

Primero: La Hermenéutica ha construido un campo de estudio centrado en la cuestión del sentido. Conforme a ello, la génesis y transmisión de sentido, esto es, la interpretación, es el proceso central a la luz del cual se analizan los aspectos fundamentales de la cultura.

Segundo: La Hermenéutica propone una instancia central para la génesis y transmisión de sentido. Sujeto, cogito, espíritu y ser humano son algunas de las caracterizaciones que se han hecho de ella.

Tercero: Con su énfasis en el sentido y en la presunta instancia central donante de sentido, la Hermenéutica ha encubierto y tergiversado los elementos no semánticos que intervienen en la generación de sentido.

Cuarto: Los dualismos de sentido y materia, sujeto y mundo material, ciencias del Espíritu y ciencias de la Naturaleza, son resultado de la operación de encubrimiento y tergiversación antes mencionada.

Quinto: El desarrollo histórico de la tergiversación ha llegado a un punto en que, mientras la Hermenéutica celebra su consolidación, queda en evidencia la tergiversación acontecida y se vuelve urgente una reconfiguración de las Ciencias del Espíritu que haga justicia a los elementos encubiertos y tergiversados.

Estos cinco planteos se apoyan, en su conjunto, sobre algunas ideas y propuestas de procedencia heideggeriana. Mencionaré las más relevantes.

En primer lugar, cabe mencionar la propuesta por la cual la ontología aporta el enfoque fundamental de estudio. Esto tiene consecuencias metodológicas importantes. Significa que la cuestión del sentido no queda fijada al nivel de la pregunta por los rendimientos cognoscitivos o por las estructuras fundamentales del conocimiento, sino que puede desarrollarse a un nivel jerárquicamente anterior respecto a los estudios sobre sujeto y objeto de conocimiento. Ese nivel previo corresponde a lo que Heidegger llama "el ser".

Un segundo planteo relevante consiste en los intentos de Heidegger por elaborar un modelo descriptivo de lo que habitualmente llamamos "ser humano" que haga justicia a su carácter relacional, situado e histórico. El término "Dasein", en las sucesivas reelaboraciones que Heidegger realiza de este, acentúa crecientemente la tensión entre elementos proyectantes o agenciales, por una parte, y elementos de facticidad, situatividad y opacidad semántica, por otra.

En tercer lugar, cabe mencionar el creciente énfasis en las instancias y dimensiones asemánticas que intervienen en la estructuración del mundo en cuanto horizonte general de sentido. Nociones como "la tierra" (Heidegger, 1977: 28) o "physis" (Heidegger, 1976: 189 sg.) y caracterizaciones 
de procesos tales como "el abrir rasgando" (Heidegger, 1989: 20) aluden a instancias inaccesibles a la comprensión y a la vez condicionantes de ella.

Un cuarto elemento a considerar consiste en la propuesta heideggeriana de un punto de vista integrador para describir el proceso general de formación de mundo, atendiendo a la tensión entre accesibilidad e inaccesibilidad de sentido y junto con ello a la tensión entre facticidad y proyección. Heidegger ensaya diversos términos para caracterizar esa perspectiva de estudio englobadora, tales como "el Ser" y "el Acontecimiento" (das Ereignis). Por su parte, tal propuesta se apoya en la idea fundamental, según la cual la dinámica de aquel proceso corresponde a lo que podríamos llamar tiempo. Esa idea es reelaborada constantemente por Heidegger, desde la investigación sobre ser y tiempo hasta los desarrollos sobre la Historia del Ser.

Por último, hay que tener en cuenta el planteo de Heidegger respecto al inevitable proceso de encubrimiento de las formaciones originarias de sentido y a la urgencia de una tarea de destrucción o desmontaje que saque a la luz los encubrimientos acontecidos y esclarezca históricamente el presente. Acompaña a este planteo el diagnóstico según el cual la dimensión de lo oculto e inaccesible ha sido el elemento constantemente tergiversado desde los inicios de la filosofía.

Como se puede advertir, los planteos heideggerianos ofrecen un marco teórico y metodológico para la estrategia compartida por Kittler y Gumbrecht. Por una parte, destacan un "más allá del sentido" (Gumbrecht, 2004: 85) que opera en los procesos formadores de sentido; por otra, ofrecen un estudio complejo de la dinámica de esos procesos -dinámica a la que Heidegger, desde los años 30, denomina "la Historia del Ser". A ello remiten expresamente tanto Gumbrecht como Kittler. Así, el primero afirma:

Una de las razones de mi decisión de esforzarme por investigar el concepto heideggeriano de ser descansa en la impresión de que ya no es suficiente constatar una y otra vez cuán hartos estamos en las Ciencias del Espíritu de un repertorio conceptual de análisis que solo nos procure acceso a la dimensión del sentido. (Gumbrecht, 2004: 98)

El único filósofo (...) que ha elaborado programáticamente durante décadas un repertorio de conceptos no metafísicos, es Martin Heidegger. (Gumbrecht, 2004: 85)

Kittler, por su parte, caracteriza a la técnica -entendida especialmente con vistas a los procesos, saberes y materiales empleados para la comunicación- como la dimensión encubierta por la Hermenéutica y las filosofías 
orientadas al sentido, y encuentra en Heidegger un referente para iluminar el proceso histórico en el cual se revertiría tal encubrimiento:

Desde Descartes a Hegel y hasta Dilthey fue el "sentido", puesto por un sujeto sobre las objetividades y medios, un obstáculo para no pensar la técnica. (Kittler, 2003: 202)

(...) solamente en Heidegger, cuando este convirtió la filosofía en "pensamiento", surgió una conciencia creciente respecto a los medios técnicos. En primer lugar, porque ya "Ser y Tiempo" tematizó lo inconspicuo de los medios cotidianos, tales como gafas y teléfonos; segundo, porque en los años treinta Heidegger describió a los medios masivos como la radio, ya no en términos existenciales, sino en términos históricos; tercero, porque después de la Segunda Guerra Mundial, Heidegger conceptualizó el comienzo de los computadores como el final fáctico de la filosofía. Ahora bien, según Heidegger, este final impulsa necesariamente (en términos de Seinsgescbichte, historia del ser) el planteo de la pregunta de por qué la lógica filosófica, tal como fue inventada por Aristóteles, finalmente condujo a su maquinización por parte de Turing, Shannon y otros. (Kittler, 2009a: 24)

Ahora bien, además de haber reconstruido la influencia de Heidegger en Kittler y Gumbrecht respecto al programa de una genealogía de las Ciencias del Espíritu (y de la Hermenéutica), cabe destacar también el gesto de apropiación creativa que estos realizan al poner a Heidegger al servicio de un planteo materialista. Las instancias asemánticas puestas de relevancia por Heidegger son entendidas ahora como "materia" o "materialidades" y el proceso general de formación de sentido es descrito principalmente con vistas al desarrollo técnico de los medios de comunicación. Este gesto de violencia interpretativa es fundamental para entender lo que hemos llamado aquí "el legado materialista" de Heidegger.

Hay por cierto diferencias entre los planteos de Gumbrecht y Kittler que merecen ser señaladas. A fin de precisar esas diferencias, conviene, en primer lugar, mencionar la radicalización del enfoque de Kittler ocurrida en el paso desde la primera fase de su pensamiento, dirigida a analizar los "sistemas de inscripción" que gestaron la literatura y la filosofía alemana del siglo XIX, hacia la segunda fase, orientada hacia los medios de comunicación y centrada en los desarrollos de las técnicas de la comunicación en el siglo $\mathrm{XX}^{15}$. La radicalización del enfoque consistió fundamentalmente en lo siguiente: mientras en la primera fase Kittler intentaba sacar a luz las condiciones materiales y técnicas de los discursos (en un procedi-

15 Para un análisis más detallado de estas fases en el pensamiento de Kittler, ver Winthrop-Young, 2005: 5 sg.; 2011: 574-576. 
miento cercano al análisis del discurso), en la segunda fase renuncia explícitamente a considerar cualquier instancia de sentido, lenguaje o discurso y propone un análisis centrado en la noción matemática y computacional de información. Su "materialismo de teoría de la información" (Kittler, 1993: 182) se propone "olvidar a los seres humanos, al lenguaje y al sentido, para dirigirnos en lugar de ello hacia las particularidades de los cinco elementos y funciones en Shannon" (Kittler, 2010: 44) ${ }^{16}$.

Conforme a lo anterior, es posible establecer una distinción entre el enfoque del primer Kittler y de Gumbrecht, por una parte, y el enfoque del segundo Kittler, por otra ${ }^{17}$. En el primer caso, la propuesta apunta a reconstruir el rol de las instancias asemánticas en la génesis de sentido. La labor programática consiste en analizar las "materialidades de la comunicación", esto es, lo "no-hermenéutico", en su relación con los procesos interpretativos (Gumbrecht) ${ }^{18}$, o bien en investigar los sistemas de notación e inscripción como condicionantes de determinados órdenes de discurso (primer Kittler, 1985: 519-522). En el segundo caso, en cambio, la propuesta se centra exclusivamente en las técnicas de producción, transmisión, registro y procesamiento de datos -es decir, en las técnicas de la información-, y elimina la noción de sentido (y junto con ello las nociones de sujeto, espíritu y ser humano) como criterio de análisis. El estudio de

16 En esta cita, Kittler hace referencia al modelo de comunicación planteado por Claude Shannon y desarrollado por Warren Weaver. Este modelo recibe una exposición canónica en el texto La teoría matemática de la comunicación (1949). La propuesta kittleriana de eliminar los modelos hermenéuticos y adoptar el modelo matemático/informático para estudiar la comunicación y la cultura tiene como base un argumento que puede reconstruirse del siguiente modo: la estructura fundamental de la comunicación consiste en los procesos de transmisión, almacenamiento y procesamiento de datos/señales y por lo tanto la cuestión focal de la que deben ocuparse los estudios sobre comunicación y cultura no es otra que la información.

17 Algunos intérpretes de Kittler, como el propio Gumbrecht (2013: 318-323), advierten una tercera y última fase en su pensamiento. Ella correspondería principalmente a los textos redactados a partir de 1995 y estaría focalizada en la música y la matemática de la antigüedad griega y su conexión con la época actual. Las diferencias, tanto en el tono como en algunos planteos específicos, respecto a la segunda fase, exceden el ámbito estricto del presente trabajo y por ello no han sido tematizadas aquí. Cabe aclarar, con todo, que algunos textos de Kittler correspondientes a la década del 2000 han sido considerados y citados aquí debido a su relevancia para el tema de la investigación. Ello demuestra, por otra parte, que las periodizaciones que ofrecen los intérpretes son más bien referenciales y que no deben entenderse como fijaciones estrictas de límites.

18 Investigar la materialidad de la comunicación significa para Gumbrecht „plantear la pregunta por las condiciones no semánticas de la génesis de sentido, es decir, por el lugar, los portadores y las modalidades de esta" (2004: 24). 
aquellas técnicas y de los escenarios post-humanos que surgen con ellas se propone ofrecer la versión actualizada de las Ciencias del Espíritu ${ }^{19}$.

A partir de la diferenciación realizada sobresalen dos planteos, uno de Gumbrecht y el otro del segundo Kittler, como dos estrategias diversas en la estela del legado materialista de Heidegger. Por una parte, Gumbrecht caracteriza la dimensión no hermenéutica en la génesis de sentido no solo en términos de "Ser", sino como "presencia". Con ello, intenta destacar el aparecer sensible y espacial y la afección sensible-corporal que este provoca: "La palabra "presencia" se refiere no solo a una relación temporal, sino también a una relación espacial con el mundo. Lo que está "presente" (...) puede afectar inmediatamente a los cuerpos humanos" (2004: 10 sg.). La tensión entre los elementos semánticos y asemánticos es caracterizada como la tensión y oscilación entre sentido y presencia, respectivamente (Gumbrecht, 2004: 68 sg., 98 sg.). En virtud de ello, Gumbrecht propone una "filosofía de la presencia" (2007: 15 sg.) centrada en el proceso de la "producción de presencia" (2007: 15 sg.). Asimismo, desarrolla la tipología de "culturas del sentido" y "culturas de la presencia", la cual permitiría sacar a la luz, en el contexto de la historia occidental, los momentos y maneras concretas en que habría emergido la dimensión no hermenéutica, encubierta y tergiversada una y otra vez por la tradición orientada hacia el sentido:

En una cultura del sentido, la forma dominante de auto-referencia humana corresponde siempre al diseño básico de lo que la cultura occidental llama "sujeto" y "subjetividad" (...). Una cultura de la presencia, en cambio, integra la existencia física y espiritual en la auto-referencia humana (piénsese, por ejemplo, en la obsesión, durante la cristiandad medieval, por la "resurrección espiritual y corporal después de la muerte"). (Gumbrecht, 2006: 319)

Por otra parte, Kittler, radicalizando su enfoque tecnicista, afirma: "Solamente es aquello que se puede encender, apagar o conectar" (1993: 182). Con ello propone que el orden fundamental de lo que hay no es sino el de las técnicas de la información. En relación con esto, Kittler ofrece una reelaboración del diagnóstico heideggeriano sobre la época técnica, es decir, sobre nuestro presente:

Por ello, el Ensamblaje [Gestell] representa no solo el "peligro", sino también (como dijo Hölderlin) "la salvación que se avecina". En efecto, las máquinas de cálculo - “computadores" en la expresión común - socavan la distinción

19 Respecto al carácter anti-humanista y post-humanista de los planteos de Kittler, ver Gane, 2005. 
que, desde la diferenciación de Aristóteles entre physis y logos, ha fundado la Metafísica misma. Ellas son ambos: lógica y física en uno solo. (Kittler 2013, 298)

El dictum heideggeriano respecto de la consumación de la metafísica en la época técnica recibe en Kittler un cambio de acento: la "salvación" y no solo "el peligro" cuenta ahora entre las potencialidades de las técnicas de la información. En este sentido, Kittler caracteriza sus estudios sobre los medios como una "Historia del Ser up to date" (2009b: 102).

\section{CONCLUSIONES}

Tras haber expuesto y analizado elementos históricos y sistemáticos de la recepción materialista y anti-hermenéutica de Heidegger, ocurrida en Alemania especialmente entre los años 80 y 90, esta provoca menos extrañeza que la que nos generó inicialmente. En efecto, lo que a primera vista podría parecer un flagrante contradicción -la recepción en clave anti-hermenéutica de un referente central de la filosofía hermenéutica-, resulta más entendible al considerar que no se trata de la recepción de Heidegger por parte de la scholarship de la Hermenéutica alemana, sino más bien del reingreso de Heidegger a Alemania desde el extranjero, en especial a través del post-estructuralismo francés. A ello se suman las características de contexto referentes al ambiente de revuelta anti-hermenéutica y al surgimiento de la ciencia de los medios. En cuanto a la dimensión temática, se trata de una recepción que ahonda en planteos operantes en cierta medida ya en el programa de Ser y Tiempo y radicalizados en el pensamiento acerca del Acontecimiento y de la Historia del Ser. Estos planteos consisten, en primer lugar, en establecer un nivel de análisis jerárquicamente anterior al conocimiento y sus estructuras, cuyo foco es el proceso por el cual surgen horizontes de sentido, esto es, el tiempo como dinámica de la constitución de mundo(s). En segundo lugar, tales planteos consisten en poner de relevancia elementos no semánticos en la génesis de sentido y en proponer un procedimiento de desmontaje (destructivo, genealógico) orientado a exponer la tensión entre elementos semánticos y asemánticos y junto con ello el encubrimiento de estos últimos, consolidado en la tradición filosófica. La figura de un Heidegger anti-humanista, crítico de la tradición filosófica y especialmente de la filosofía moderna del sujeto, se enfatiza a partir de tal recepción.

Ahora bien, hay otros aspectos de dicha recepción que no pueden entenderse como aproximaciones selectivas y estratégicas al pensamiento de Heidegger, sino más bien como intentos por corregir y reorientar las propuestas de este. Allí, el legado materialista de Heidegger alcanza sus límites 
y se delinean propuestas materialistas que no se pueden reconducir hacia Heidegger. Ejemplos de ello son, por una parte, la orientación hacia las técnicas de la comunicación y la consideración de estas como instancias superadoras de los dualismos de la tradición filosófica en un escenario post-humanista (Kittler, 2004: 250; 2009a: 23, 29), y, por otra parte, la propuesta tendiente a equiparar los elementos asemánticos en la genésis de sentido con la presencia física en cuanto afección sensible-corporal provocada por las cosas del mundo cotidiano (Gumbrecht ${ }^{20}$.

En atención a los límites en la influencia de Heidegger, cabe señalar lo siguiente. En primer lugar, los mencionados intentos por corregir y aggiornar el pensamiento de Heidegger se encuadran en el marco de las ciencias de la cultura, con una especial orientación hacia la mediación comunicativa en cuanto instancia fundamental. El pensamiento de Heidegger, con sus filosofemas centrales (ser, Dasein, Historia del Ser, etc.), queda resituado en el eje de ciencias de los medios y ciencias de la cultura ( $M e$ dienwissenschaften - Kulturwissenschaften). Es una pregunta abierta si tal desplazamiento hace justicia al lugar sistemático que tiene para Heidegger la indagación sobre el ser, sea como ontología fundamental, o bien como el pensar acerca del Acontecimiento (Ereignis-Denken).

En segundo lugar, en el materialismo anti-hermenéutico la noción de "materia" es asumida estratégicamente como clave para superar el dualismo físico-espiritual de la tradición. El ejemplo más radical de esta estrategia es el monismo materialista-tecnicista del segundo Kittler, centrado en la información. Ahora bien, la acepción materialista de la información, esto es, la noción de información como diferencia reiterable de estados físicos básicos ${ }^{21}$, parece suponer una ontología de lo que está ahí delante (Vorbandenes), por lo cual quedaría expuesta directamente a las objeciones de Heidegger.

20 Ver Gumbrecht, 2004: 85-98. Gumbrecht interpreta la noción de "Ser" en Heidegger (tanto en "Ser y Tiempo" como en escritos de los años 30 y 40) como el carácter de presencia constitutivo del aparecer sensible y espacial de las cosas, opuesto por tanto a la dimensión conceptual y del sentido en general. "Ser no es sentido. Pertenece a la dimensión de las cosas (...) Tiene sustancia y por ello (...) ocupa lugar en el espacio" (2004: 88). Es tanta la discrepancia de este planteo con otras interpretaciones, como por ejemplo la lectura trascendentalista (véase Crowell, 2001: 167-221) y especialmente con planteos explícitos de Heidegger (por ejemplo, aquellos respecto a la diferencia entre ser y ente), que cabe pensar que si bien Gumbrecht presenta esto como una lectura de Heidegger, se trata más bien de un intento por corregir y reorientar a Heidegger a partir de sus propios textos.

21 Ver Gane \& Sale, 2007: 325 sg. Véase también la siguiente afirmación de Gane: "La información ya no es tratada [por Kittler / R.R.] puramente como una función probablística (como lo fue para Shannon y Weaver), sino como una propiedad material que no se distingue de los componentes físicos que la hacen posible” (2005: 29).

VERITAS, N $^{\circ} 44$ (diciembre 2019) 
Hechas estas observaciones críticas, cabe preguntarse cómo hay que acoger la recepción de Heidegger aquí analizada. Una aproximación fructífera hacia tales planteos consiste en entenderlos como una herencia autónoma, con fines propios. Corresponde asumirlos como parte del desafío de pensar el presente. En otras palabras, su ámbito es el de una ontología del presente y no el de la filología de Heidegger. Vistos desde esa perspectiva, su impertinencia puede resultar sin duda productiva.

\section{REFERENCIAS}

Burkhardt, M. y Sandbothe, M. (2014). Medienphilosophie. En J. Schröter (Ed.), Handbuch Medienwissenschaft (pp. 377-383). Stuttgart: Metzler.

Busch, Ch. (2014). Strategische Zitaten. Zu Friedrich Kittlers Heidegger-Lektüre. Zeitschrift für Literaturwissenschaft und Linguistik, 44(3), 161-169.

Crowell, St. (2001). Husserl, Heidegger, and the Space of Meaning. Evanston: Northwestern University Press.

Dillet, B. et al. (Eds.) (2013). The Edinburgh Companion to Poststructuralism. Edimburgo: Edinburgh University Press.

Enzensberger, H. M. (1982). Constituents of a Theory of the Media. En H. M. Enzensberger, Critical Essays (pp. 46-76). New York: Continuum.

Frank, M. (2004). Integración sin entusiasmo. La filosofía alemana entre la asimilación y el rechazo de la tradición. Revista de Occidente. Pensar en alemán hoy. Vuelve la Ilustración, (282), 5-14.

Frank, M. (2011). ¿Qué es el neoestructuralismo? México: FCE.

Gadamer, H.-G. (1986). Wahrheit und Methode. Gesammelte Werke, Tomo 1. Tubinga: Mohr Siebeck. (Verdady Método. Salamanca: Sígueme, 1999).

Gane, N. (2005). Radical Post-Humanism. Friedrich Kittler and the Primacy of Technology. Theory, Culture \& Society, 22(3), 25-41.

Gane, N. \& Sale, St. (2007). Interview with Friedrich Kittler and Mark Hansen. Theory, Culture \& Society, 24(7-8), 323-329.

Garnica, N. (2018). Asimilación y rechazo de la filosofía francesa en Alemania. La recepción de Manfred Frank: romanticismo, hermenéutica y deconstrucción. Cuestiones de Filosofía, 4(22), 37-67.

Gumbrecht, H. \& Pfeiffer, K. (1988). Materialität der Kommunikation. Fráncfort: Suhrkamp.

Gumbrecht, H. (2003). Production of Presence. Stanford: Stanford University Press. (Diesseits der Hermeneutik. Die Produktion von Präsenz. Fráncfort: Suhrkamp, 2004).

Gumbrecht, H. (2006). Presence achieved in language. (With special attention given to the presence of the past). History and Theory, 45(3), 317-327.

Gumbrecht, H. (2007). De la Hermenéutica edípica a la Filosofía de la presencia. Fractal, (47), 15-40.

Gumbrecht, H. (2012). Präsenz: Berlin: Suhrkamp. 
Habermas, J. (1970). Der Universalitätsanspruch der Hermeneutik. En R. Bubner, R. et al. (Eds.) Hermeneutik und Dialektik. Hans-Georg Gadamer zum 70.Geburtstag. Tomo 1. (pp. 73-103). Tubinga: Mohr Siebeck, 1970.

Habermas, J. (1985). La modernidad, un proyecto incompleto. En H. Foster (Ed.). La posmodernidad (pp. 19-36). Barcelona: Kairós.

Habermas, J. (1993). El discurso filosófico de la modernidad. Madrid: Taurus.

Heidegger, M. (1976). Wegmarken. Fráncfort: Klostermann. (Hitos. Madrid: Alianza, 2001).

Heidegger, M. (1977). Holzwege. Fráncfort: Klostermann. (Caminos de bosque. Madrid: Alianza, 2012).

Heidegger, M. (1989). Vom Ursprung des Kunstwerks. Erste Ausarbeitung. Heidegger Studien, 5, 5-22. (Del origen de la obra de arte. Primera versión. Revista de Filosofía, 115 (2016), 11-36).

Kittler, F. (Ed.) (1980). Austreibung des Geistes aus den Geisteswissenschaften. Paderborn: Schöningh.

Kittler, F. (1985). Aufschreibesysteme 1800 / 1900. Múnich: Fink. (Discourse Networks 1800 / 1900. Stanford: Stanford University Press, 1990).

Kittler, F. (1993). Draculas Vermächtnis. Fráncfort: Reclam.

Kittler, F. (1999). Gramophone, Film, Typewriter. Stanford: Stanford University Press.

Kittler, F. (2000). Eine Kulturgeschichte der Kulturwissenschaft. Múnich: Fink.

Kittler, F. (2009a). Towards an Ontology of Media. Theory, Culture \& Society, 26(23), 23-31.

Kittler, F. (2009b). Das kalte Modell von Struktur. Zeitschrift für Medienwissenschaft, 1, 93-102.

Kittler, F. (2010). Optical media. Cambridge: Polity Press.

Kittler, F. (2012). Vorwort zu Aufschreibesysteme 1800 1900. Zeitschrift für Medienwissenschaft, 6/I, 117-126.

Kittler, F. (2013). The Truth of the Technological World. Stanford University Press.

Mersch, D. (2006). Medientheorien zur Einführung. Hamburgo: Junius.

Michelfelder, C. \& Palmer, R. (Eds.) (1989). Dialogue and Deconstruction. The Gadamer-Derrida Encounter. New York: New York University Press.

Pias, C. (Ed.). (2012). Aufschreibesysteme 1980/2010, Zeitschrift für Medienwissenschaften, 6(1), 114-192.

Shannon, C. \& Weaver, W. (1949). The Mathematical Theory of Communication. Chicago: The University of Chicago Press.

Vattimo, G. (1991). Hermenéutica: nueva koiné. En G. Vattimo. Ética de la Interpretación (pp. 55-72). Buenos Aires: Paidós.

Wellbery, D. (1990) Foreword. En F. Kittler. Discourse Networks 1800 / 1900 (pp. vii-xxxiii). Stanford: Stanford University Press.

Winthrop-Young, G. (2005). Friedrich Kittler zur Einführung. Hamburgo: Junius.

Winthrop-Young, G. (2011). Friedrich Kittler: Kultur als Datenverarbeitungsgestell. En St. Moebius (Ed.). Kultur. Theorien der Gegenwart (pp. 572-579). Wiesbaden: Springer. 
Winthrop-Young, G. \& Wutz, M. (1999). Translator's Introduction. En F. Kittler. Gramophone, Film, Typewriter (pp. xi-xxxviii). Stanford: Stanford University Press.

Winthrop-Young, G. \& Gane, N. (2006). Friedrich Kittler: An Introduction. Theory, Culture \& Society, 23(7-8), 5-16.

Sumario: Introducción; 1. La recepción materialista y anti-hermenéutica de Heidegger en contexto; 2. Aspectos temáticos fundamentales; Conclusiones; Referencias. 